\title{
ANDREAAOKI COSTA
}

Atendimento dos enfermeiros no tratamento do lúpus eritematoso sistêmico juvenil: um recorte nacional

Dissertação apresentada a Faculdade de Medicina da Universidade de São Paulo para obtenção do titulo de

Mestre em Ciências da Saúde

Programa de Pediatria

Orientadora: Prof(a). Dr(a). Juliana Caires de Oliveira Achili Ferreira

São Paulo

2021 


\section{ANDREAAOKI COSTA}

Atendimento dos enfermeiros no tratamento do lúpus eritematoso sistêmico juvenil: um recorte nacional

Dissertação apresentada a Faculdade de Medicina da Universidade de São Paulo para obtenção do titulo de Mestre em Ciências da Saúde

Programa de Pediatria Orientadora: Prof(a). Dr(a). Juliana Caires de Oliveira Achili Ferreira

(Versão corrigida. Resolução CoPGr 5890, de 20 de dezembro de 2010. A versão original está disponível na Bibiloteca FMUSP)

São Paulo

2021 
Dados Internacionais de Catalogação na Publicaçăo (CIP)

Preparada pela Biblioteca da

Faculdade de Medicina da Universidade de Săo Paulo

Creproduçăo autorizada pelo autor

Costa, Andrea Aoki

Atendimento dos enfermeiros no tratamento do lúpus eritematoso sistêmico juvenil : um recorte nacional / Andrea Aoki Costa. -- São Paulo, 2021.

Dissertaçăo (mestrado)--Faculdade de Medicina da Universidade de Săo Paulo.

Programa de Pediatria.

Orientadora: Juliana Caires de Oliveira Achili Ferreira.

Descritores: 1.Lúpus eritematoso sistêmico 2.Adolescente 3.Enfermeiro 4.Planejamento de assistência ao paciente 5 . Enfermagem pediátrica 6 . Consulta de enfermagem

$\mathrm{USP} / \mathrm{FM} / \mathrm{DBD}-232 / 21$ 


\section{Agradecimentos}

Primeiramente agradeço à Deus, pela preseça constante durante a minha vida. Obrigada, por ter abençoado dias difíceis com momentos inesquecíveis e pessoas especiais. Obrigada por tornar possível a concretização deste sonho.

A minha família: irmãos, cunhadas, sobrinhos, sobrinhas e sobrinha neta e em especial meus pais, Geni e Almir (in memoriam), por terem ensinados valores sobre a vida e sempre apoiarem as minhas escolhas.

À minha querida orientadora Dra. Enf ${ }^{a}$ Juliana Caires de Oliveira Achili Ferreira, por ter me ajudado no desenvolvimento e conclusão deste trabalho. Obrigada por acreditar no meu potencial, por compartilhar conhecimento e experiência acerca dos pacientes com lúpus e por fazer parte da construção da minha carreira.

Ao Prof. Dr. Clovis Artur Almeida da Silva, uma pessoa de tamanha bondadee alegria que Deus colocou no meu caminho no tempo certo. Obrigada por acreditar no meu potencial e compartilhar seu conhecimento e experiência relacionada ao paciente com lúpus.

À Divisão de Enfermagem do Instituto da Criança pelas oportunidades de crescimento profissional. Em especial a minha supervisora de serviço hospitalar Maria Socorro Soares Silva, que desde o início se prontificou a fazer o necessário para permitir a conclusão deste trabalho.

À Mariza Kazue Umetsu Yoshikawa, pelo apoio e prontidão constantes com que sempre trabalha.

À Mônica Alves de Souza pela dedicação e eficiência no trabalho que desenvolve. 


Esta dissertação está de acordo com as seguintes normas, em vigor no momento
desta publicação:
Referências: adaptado de International Committe of Medical Journals Editors
(Vancouver).
Universidade de São Paulo. Faculdade de Medicina: Divisão de Biblioteca e
Documentação. Guia de apresentação de dissertações, teses e monografias.
Elaborado por Anneliese Carneiro da Cunha, Maria Julia de A. L. Freddi, Maria F.
Crestana, Marinalva de Souza Aragão, Suely Campos Cardoso, Valeria Vilhena. 3a
ed. São Paulo: Divisão de Biblioteca e Documentação; 2011.
Abreviatruras dos titulos dos periódicos de acordo com List of Journals Indexed in
Index Medicus.




\section{Sumário}

LISTA DE TABELAS

LISTA DE ANEXOS:

RESUMO

\section{ABSTRACT}

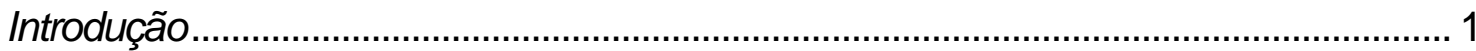

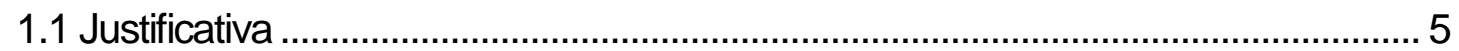

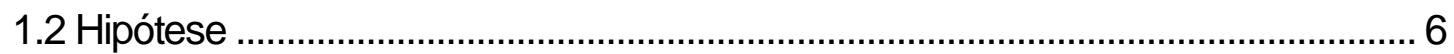

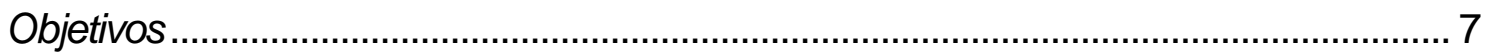

2.1 Geral:

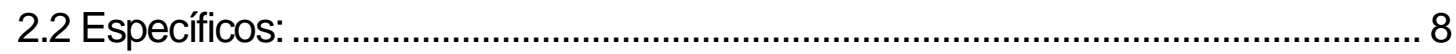

Método

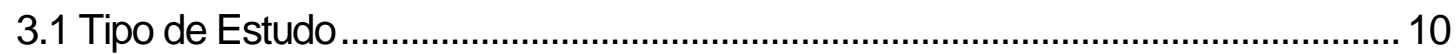

3.2 População de Estudo ....................................................................................... 10

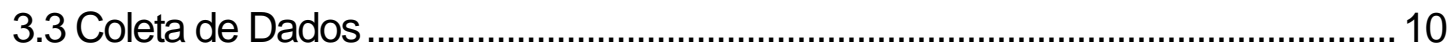

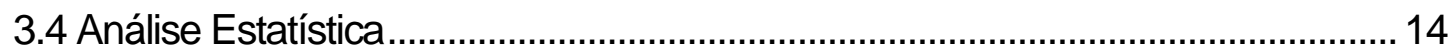

3.5. Aspecto ético e legal........................................................................................ 15

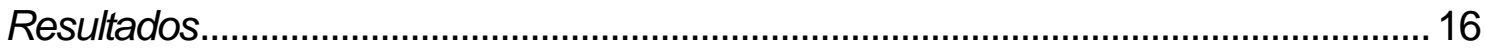

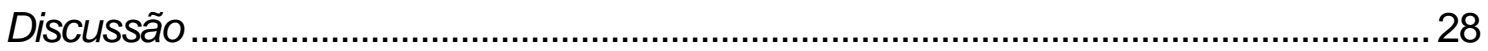

Conclusão

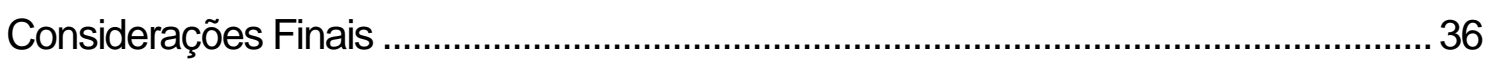

Anexos 


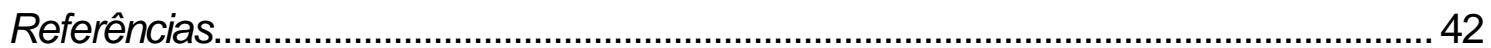




\section{LISTA DE TABELAS:}

Tabela 1: Dados demográficos e caracterização dos enfermeiros

Tabela 2: Caracterização do atendimento dos profissionais

Tabela 3: Conhecimento dos enfermeiros sobre as ferramentas para avaliar o LESJ

Tabela 4: Procedimentos e Terapêutica disponíveis aos pacientes com LESJ .........21

Tabela 5: Práticas para melhor assistência aos pacientes com LESJ

Tabela 6: Atendimento exclusivo do serviço público versus serviço privado. 24

Tabela 7: Análise entre profisisonais que já cuidaram de pacientes com LESJ. 25

Tabela 8: Análise entre profissionais que atenderam pacientes com LESJ

hospitalizados. 26

Tabela 9: Profissionais que trabalham em serviço e universidade pública x serviço público 


\section{LISTA DE ANEXOS:}

Anexo 01: Questionário de avaliação do atendimento dos enfermeiros aos pacientes

com Lupus Eritematosos Sistêmico Juvenil 38 


\section{RESUMO}

Costa AA. Atendimento dos enfermeiros no tratamento do lúpus eritematoso sistêmico juvenil: um recorte nacional [dissertação]. São Paulo: Faculdade de Medicina, Universidade de São Paulo; 2021.

Introdução: O lúpus eritematoso sistêmico juvenil (LESJ) é uma doença multissistêmica crônica de etiologia multifatorial e incidência considerável na América Latina. Caracteriza-se por alterações do sistema imunológico e produção de múltiplos anticorpos, com nítida predileção pelo sexo feminino e início na infância ou adolescência em 15 a $20 \%$ dos casos. A complexidade do tratamento LESJ envolve vários aspectos, como conhecimento da doença e aderência ao tratamento por parte do paciente e família, disponibilidade de exames e medicamentos, atividade ou remissão da doença, que pode se manifestar de diferentes formas. Recursos humanos capacitados com conhecimento da doença são de suma importância no diagnóstico precoce e encaminhamento aos setores de referência, e o enfermeiro, profissional que atua diretamente no cuidado ao paciente desde atenção básica até hospitais de alta complexidade, quando conhece os sinais clínicos e as manifestações do LESJ, as ferramentas disponíveis para a doença e utiliza instrumentos de rotina, como questionários de adesão, dor e qualidade de vida, pode atuar desde o auxílio do diagnóstico precoce até o tratamento. Objetivos: Avaliar a prevalência de atendimento de crianças e adolescentes com LESJ por enfermeiros brasileiros e descrever as práticas de cuidados; Identificar associação entre dados demográficos dos enfermeiros brasileiros e conhecimento do LESJ; Descrever a frequencia do uso de ferramentas para diagnóstico e tratamento do LESJ por enfermeiros brasileiros. Método: estudo transversal analítico realizado a partir das respostas de um questionário auto-aplicável sobre o atendimento dos enfermeiros brasileiros aos pacientes com LESJ entre agosto de 2018 e setembro de 2019. Resultados: foram respondidos 111/373 (29,4\%) questionários, provenientes das regiões Sul, Sudeste, Centro-Oeste e Nordeste. A maioria dos profissionais é do sexo feminino $(90,1 \%)$, trabalha no senviço público $(82,9 \%)$, não utiliza $(51,4 \%)$ ou desconhece $(37,8 \%)$ as ferramentas utilizadas para 
avaliar a atividade da doença e acredita que o conhecimento da doença é uma prática que precisa ser melhorada (83,3\%). Entre os cuidados gerais disponíveis aos pacientes com LESJ prevalece a abordagem pela equipe multidisciplinar (65,8\%), enquanto que hospitalização (45,9\%) e má aderência medicamentosa $(27,9 \%)$ foram os problemas mais citados pelos profissionais, além do desconhecimento do processo de transição institucional para o cuidado adulto por metade dos profissionais (50,5\%). Foram realizadas comparações dos cuidados esperados aos pacientes quanto ao tipo de serviço, cuidado ao paciente hospitalizado e cuidado do paciente com LESJ, sem diferenças estatisticamente significantes. Conclusões: Os profissionais participantes realizam os cuidados de rotina aos pacientes mas não utilizam ferramentas para avaliar a atividade da doença nem ferramentas auxiliares ao tratamento, além de atividades esperadas aos pacientes com LESJ, como verificar caderneta de vacinação, orientar proteção solar, questões relacionadas à aderência, transição ao cuidado adulto, cuidado paliativo, o que pode estar relacionado à lacuna de conhecimento, um fator comum mencionado pela maioria dos profissionais, corroborando a necessidade de disseminação da informação e capacitação para o melhor tratamento.

Descritores: Lúpus eritematoso sistêmico; Adolescente; Enfermeiro; Planejamento de assistência ao paciente; Enfermagem pediátrica; Consulta de enfermagem. 


\section{ABSTRACT}

Costa AA. Care provided by nurses to patients with juvenile systemic lupus erythematosus: a partial view [dissertation]. São Paulo: "Faculdade de Medicina, Universidade de São Paulo"; 2021.

Introduction: Juvenile Systemic Lupus Erythematosus (JSLE) is a chronic multisystemic disease of multifactorial etiology and considerable incidence in Latin America. It is characterized by changes in the immune system and production of multiple antibodies, with clear predilection for females and onset in childhood or adolescence in 15 to $20 \%$ of cases. The complexity of JSLE treatment involves several aspects, such as knowledge of the disease and adherence to treatment by the patient and family, availability of tests and medications, activity or remission of the disease, which may manifest itself in different ways. Human resources trained with knowledge of the disease are of paramount importance in early diagnosis and referral to the reference sectors, and the nurse, a professional who acts directly in the care of patients from primary care to hospitals of high complexity, when he knows the clinical signs and manifestations of JSLE, the tools available for the disease and uses routine instruments, such as assessment questionnaires, pain and quality of life, can act from the aid of early diagnosis to treatment. Objectives: Assess the prevalence of care for children and adolescents with JSLE by Brazilian nurses and describe care practices; Identify the association between demographic data of Brazilian nurses and knowledge of the LESJ; Describe the frequency of use of tools for the diagnosis and treatment of JSLE by Brazilian nurses. Method: analytical study, which was performed from the answers of a self-administered questionnaire, the care provided by Brazilian nurses to patients with JSLE between august 2018 to october 2019. Results: 111/373 (29.4\%) questionnaires from the South, Southeast, Midwest and Northeast regions. Most professionals are female $(90.1 \%)$, work in the public service $(82.9 \%)$, do not use $(51.4 \%)$ or unaware $(37.8 \%)$ the tools used to evaluate the activity of the disease and believes that knowledge of the disease is a practice that needs to be improved (83.3\%). Among the general care available to patients with JSLE, the multidisciplinary team approach $(65.8 \%)$ and poor drug adherence (27.9\%) were the problems most mentioned by professionals, besides the ignorance of the process of institutional transition to adult care 
by half of the professionals (50.5\%). Comparisons of professionals on expected care of patients regarding the type of service, hospitalized patient care, and care provided to patients with JSLE did not reveal statistically significant differences. Conclusions: The participating professionals perform routine care to patients but do not use tools to evaluate disease activity or support tools for treatment, in combination with activities expected to patients with JSLE, such as checking vaccination booklet, recomending sun protection, issues related to adherence, transition to adult care, palliative care, which may be related to the knowledge gap, a common factor mentioned by most professionals, corroborating the need for dissemination of information and training for better treatment.

Descriptors: Lupus erythematosus systemic; Adolescent; Nurse; Patient care planning; Pediatric nursing; Nursing assessment. 
Introdução 


\section{Introdução}

\section{Lúpus Eritematoso Sistêmico Juvenil}

O lúpus eritematoso sistêmico juvenil (LESJ) é uma doença multissistêmica crônica de etiologia multifatorial que envolve herança multigênica, fatores ambientais ${ }^{1}$, caracterizada por alterações do sistema imunológico e produção de múltiplos anticorpos. $^{2.5}$

É uma doença rara, com incidência anual nos Estados Unidos estimada em 0,4 a 0,6 casos/100.000 crianças e adolescentes, ${ }^{6}$ sendo excepcional antes dos cinco anos de idade. ${ }^{7}$ Estima-se que 15 a 20\% dos casos de LES têm início durante a infância ou adolescência, com nítida predileção pelo sexo feminino, sendo que a proporção de sexo feminino:masculino varia de 4:3 durante a primeira década de vida a 9:1 durante segunda década de vida. ${ }^{8-9}$

As manifestações clínicas do LESJ são extremamente variáveis dependendo do órgão ou sistema acometido. Os acometimentos mucocutâneo, principalmente eritema malar, fotossensibilidade e úlceras de mucosa oral e nasal ${ }^{10-14}$, renal ${ }^{15,16} \mathrm{e}$ músculo esquelético, como artrite aguda não erosiva e artralgia ${ }^{17}$, são bastante comuns.

Um estudo realizado em 2015 pelo Grupo Latino Americano de Estudo do Lupus (GLADEL) comparou 230 pacientes com LESJ e 984 pacientes com Lupus Eritematoso Sistêmico Adulto e verificou que o LESJ tem apresentação mais grave, os pacientes tem maior atividade da doença, comprometimento hematológico, cutâneo e de sistema nervoso central. É mais frequente em africanos latino-americanos e mestiços, os pacientes apresentam mais critérios definidores da doença e em maior número. A mediana de idade verificada no estudo referente ao início do LESJ foi de 15,3 anos e a mediana de idade no diagnóstico foi de 16,4 anos. ${ }^{18}$

O outro grupo latinoamericano que estuda o Lúpus é o Grupo Brasileiro de Lupus Juvenil, que já realizou estudos dividindo os pacientes em três grupos denominados Grupo A (pacientes de início precoce menores de seis anos de idade), Grupo B (pacientes de idade escolar entre seis e doze anos) e Grupo C (adolescentes maiores de doze até dezoito anos de idade). Um estudo de coorte observacional foi realizado em dez centros incluindo 847 pacientes com LES e evidenciou que o grupo de início precoce teve desfechos distintos e mais graves, e os pacientes tiveram doença multissistêmica com alta frequência de envolvimento de órgãos principais e 
escores elevados de SLEDAI-2K em todos os grupos etários, sendo a mortalidade um desfecho precoce, atribuída as infecções associadas a atividade da doença. ${ }^{19}$ Outro estudo do grupo realizado com 1555 pacientes dividiu os mesmos em grupos baseados no intervalo os primeiros sintomas e o diagnóstico da doença, evidenciou o diagnóstico tardio para a maioria dos pacientes, provavelmente devido ao início leve da doença, e que a minoria dos pacientes teve intervalo de tempo muito curto entre diagnóstico e apresentação com condição multissistêmica mais grave e ativa. ${ }^{20}$

Além disso, as infecções agudas/crônicas e sepse são uma importante causa de morbidade e mortalidade no LESJ ${ }^{21-22}$, causadas mais frequentemente por vírus como herpes-zoster, bactérias e menos comumente por agentes oportunistas, como tuberculose e fungo ${ }^{23-27}$.

Vários estudos multicêntricos de pacientes com LESJ seguidos no Estado de São Paulo foram publicados e incluíram participação de profissionais de saúde, particularmente médicos e enfermeiros. Dentre estes, destacaram-se: manifestações iniciais ao diagnóstico ${ }^{19}$, infecções por herpes-zoster e fúngicas ${ }^{28-29}$, pancreatite ${ }^{30}$, urticária $^{31}$, síndrome de Evans $^{32}$, uveíte ${ }^{33}$, artrite crônica ${ }^{34}$, autoanticorpos ${ }^{35}$ e prognóstico ${ }^{36}$. Entretanto, o caráter retrospectivo destes estudos, com alguns dados faltantes nos prontuários, particularmente relacionados ao tratamento, e a realização apenas nos centros de São Paulo contém limitações.

\section{O paciente com LESJ}

O caráter crônico do LESJ, somado às eventuais complicações e possíveis sequelas ou limitações, demanda uma atenção especial ao paciente e sua família.

O paciente pode apresentar sintomas limitantes como artrites, dor ou inflamações que ocasionam febre e alterações laboratoriais, além de necessidade de hospitalização, o que caracteriza a atividade da doença. O tratamento farmacológico é necessário para os órgãos e sistemas acometidos, visando a resolução do quadro clínico e aquisição da fase de remissão da doença; todavia, o uso de corticóides, imunossupressores, antimaláricos e antiinflamatórios, podem desencadear reações como face cushingoide, alopécia, desconforto gástrico, e interferirem na aderência medicamentosa, especialmente em adolescentes.

Algumas condutas como a necessidade de uso contínuo de protetor solar, uso de medicamentos mesmo que em doses baixas e realização de atividade física e/ou fisioterapia fazem parte da vida da maioria dos pacientes que estão com a doença 
controlada, ou em remissão, e devem ser avaliadas quando a efetividade e adesão adequada.

Ademais, os sintomas cutâneos como úlceras orais e nasais causam dor e desconforto, enquanto que o eritema malar, conhecido também como asa de borboleta, gera muitas vezes dificuldade de aceitação da própria imagem, potencializada quando acompanhada de alopécia, e devem ser avaliados com atenção.

\section{O enfermeiro no tratamento do paciente com LESJ}

A complexidade do tratamento LESJ envolve vários aspectos, como conhecimento e entendimento da doença, aderência ao tratamento por parte do paciente e família, disponibilidade de exames e medicamentos, estágio de atividade ou remissão da doença, que pode se manifestar de diferentes formas. A idade dos pacientes também deve ser considerada, pois quando adolescentes, têm aspectos oriundos deste período de crescimento e desenvolvimento que afetam diretamente 0 tratamento e a qualidade de vida.

Um estudo realizado com médicos reumatologistas atuantes na América Latina revelou a necessidade destes em trabalhar com equipe multiprofissional e de treinamento dos profissionais sobre tratamento do LESJ, pois esta doenca tem incidência considerável na América Latina quando comparada aos demais continentes, e sua complexidade exige, além de recursos materiais, recursos humanos capacitados para o diagnóstico precoce e encaminhamento aos setores de referência ${ }^{37}$.

Nessa temática, é de suma importância que o enfermeiro, profissional que atua diretamente no cuidado ao paciente desde atenção básica até hospitais de alta complexidade, conheça os sinais clínicos e as manifestações do LESJ, ferramentas disponíveis para a doença e utilize instrumentos de rotina, como questionários de adesão, dor e qualidade de vida, podendo atuar desde o auxílio do diagnóstico precoce até $\mathrm{o}$ tratamento. Entre as ferramentas encontram-se o Índice de danos cumulativo SLICC/ACR (Systemic Lupus Erythematosus Collaborating Clinics/American College of Rhumatology Damage Index $)^{38}$ e o Índice de atividade da doença do lúpus eritematoso sistêmico 2000 (SLEDAI-2K) ${ }^{39}$, que avaliam dano cumulativo e atividade da doença, respectivamente e, embora sejam utilizados majoritamente pelo médico na consulta, contêm aspectos que o enfermeiro, ao realizar a consulta de enfermagem, consegue identificar e sinalizar à equipe. Entre eles existe a febre, alopécia, eritema, úlceras orais, 
e artrites, já citados anteriormente, além de alterações urinárias, como leucocitúria, proteinúria e cilindrúria, alterações no hemograma como leucopenia e linfopenia, vasculite, cefaléia, miosite caracterizada por fraqueza ou dor muscular. Sinais e sintomas do sistema nervoso também podem ser percebidos na consulta: paciente pode referir cefaléia importante, convulsão, psicose, ou até mesmo diálogo incoerente, dificuldade de manter atenção e distúrbio de percepção, que constituem a síndrome cerebral orgânica.

É esperado que a consulta de enfermagem ${ }^{40}$ em nível ambulatorial, ou quando realizada ao paciente hospitalizado, contemple, além da mensuração dos sinais vitais e dados antropométricos, um bom histórico de enfermagem com anamnese e exame físico minunciosos. A condução da consulta baseada nos achados iniciais, somada ao conhecimento de doença e raciocínio clínico, deve considerar aspectos importantes como aderência medicamentosa, de tratamento, avaliação de dor, qualidade de vida.

Para tanto, faz-se necessário conhecer o cenário atual de enfermeiros, em nível nacional, quanto ao conhecimento e atendimento de pacientes com LESJ, e a partir daí traçar estratégias, mediante necessidade, que visem um atendimento padronizado contemplando o tratamento com enfoque especial nos pacientes adolescentes, faixa etária mais acometida, cujo período de mudanças no crescimento e desenvolvimento afetam diretamente a aceitação da doença e a aderência ao tratamento.

A utilização de questionários auto-aplicáveis tem sido uma ferramenta importante e de fácil acesso para avaliar epidemiologia e tratamento das doenças crônicas, como o LESJ. Entretanto, ainda não foi utilizada para avaliar estes aspectos sistematicamente nos enfermeiros brasileiros que acompanham pacientes com LESJ.

\subsection{Justificativa}

As experiências dos enfermeiros, são fundamentais no reconhecimento, seguimento e adesão aos diversos tratamentos realizados nos pacientes com LESJ, uma doença incapacitante quando não tratada adequadamente, que pode causar sequelas nos pacientes, e é imprescindível que as mesmas sejam divulgadas e disponibilizadas à classe científica. 
1.2 Hipótese

A disponibilidade do uso de ferramentas na prática clínica diária, bem como o conhecimento e a atuação dos profissionais e tratamentos disponíveis para o LESJ, podem ser diferentes nos diversos serviços de Pediatria do Brasil. 
Objetivos 


\section{Objetivos}

2.1 Geral:

1. Avaliar a prevalência de atendimento de crianças e adolescentes com LESJ por enfermeiros brasileiros e descrever as práticas de cuidados.

2.2 Específicos:

1. Identificar associação entre dados demográficos dos enfermeiros brasileiros e conhecimento do LESJ;

2. Descrever a frequencia do uso de ferramentas para diagnóstico e tratamento do LESJ por enfermeiros brasileiros. 
Método 


\section{Metodologia}

\subsection{Tipo de Estudo}

Trata-se de um estudo transversal analítico realizado a partir das respostas de um questionário auto-aplicável, o atendimento dos enfermeiros brasileiros aos pacientes com LESJ.

\subsection{População de Estudo}

A população do estudo foi composta por uma amostra de conveniência que inclui os enfermeiros pediátricos do Brasil que já cuidaram de pacientes com LESJ.

Para compor esta amostra foram enviados e-mails aos médicos reumatologistas do Brasil $(n=160)$ que atuam em instituições públicas e privadas, membros da Sociedade Brasileira de Reumatologia Pediátrica, informando sobre o estudo e solicitando que os mesmos disponibilizassem os e-mails dos profissionais enfermeiros membros de suas equipes.

Devido ao número pequeno de reumatologistas pediátricos brasileiros e caráter raro da doença, também foram entregues questionários escritos aos enfermeiros que já cuidaram de pacientes com LESJ durante o evento da Sociedade Brasileira de Enfermeiros Pediatras - SOBEP ocorrido em 2019.

\subsection{Coleta de Dados}

O questionário auto-aplicável foi composto por itens que buscam caracterizar os participantes e avaliar o atendimento dos pacientes pediátricos portadores de lúpus. Contém questões de múltipla escolha-resposta e dicotômica (sim e não). O tempo estimado para respostas foi de aproximadamente 15 minutos.

Este questionário foi disponibilizado na ferramenta REDCap, um aplicativo gratuito e seguro disponível na Web, projetado para suportar a captura de dados para estudos de pesquisa. O sistema foi desenvolvido por um consórcio multi-institucional iniciado na Universidade de Vanderbilt. A coleta de dados é personalizada para cada estudo ou ensaio clínico pela equipe de pesquisa com orientação do pessoal de apoio da Harvard Catalyst EDC. ${ }^{40}$ Este instrumento é disponível para o HC-FMUSP no site https://redcap.hc.fm.usp.br. A primeira página do questionário explica sobre a 
realização da pesquisa, fornece os dados de aprovação da mesma e da Comissão de Ética e explica sobre confidencialidade dos dados e não obrigatoriedade de participar, não sendo solicitados dados pessoais como nome e local de trabalho.

Os questionários entregues durante o evento da SOBEP foram impressos do sistema REDCAP e as respostas adicionadas no próprio sistema para compor a análise. Como o questionário náo solicita nome nem e-mail ou outra fonte de identificação dos profissionais, a identidade dos mesmos foi preservada e os dados analisados sem comprometimento ético.

A pesquisa eletrônica incluiu questões relacionadas aos seguintes tópicos:

1. Dados demográficos dos profissionais [(gênero, anos de prática, país), localização da prática profissional (prática de universidade pública, prática de universidade privada, prática clínica pública e/ou prática clínica privada), unidade de trabalho (internação, ambulatório, hospital dia, UTI, PS, UBS), realização de consulta de enfermagem, trabalho com outros profissionais]

2. Emprego dos profissionais (tempo parcial ou integral).

3. Disponibilidade de seguro de saúde para pacientes com LESJ (seguro de saúde pública, seguro privado e/ou outro).

4. Realizou Consulta de Enfermagem (CE), também denominada Processo de Enfermagem, antes ou após a consulta médica. A CE é uma atividade exclusiva do enfermeiro. Nesse sentido, percebe-se que haverá, na CE, uma tríade presente: o levantamento de dados, a análise e o plano de cuidados, os quais podem ser desenvolvidos em quatro passos: avaliação, identificação de problemas e/ou diagnóstico de enfermagem, intervenção ou implementação e evolução. $^{41}$

5. Conhecimento dos critérios de classificação para o diagnóstico de LESJ: Critérios do Colégio Americano de Reumatologia (ACR) de 1997 e/ou dos Critérios do Systemic Lupus International Collaborating Clinics (SLICC) de $2012^{38}$.

6. Utilização de ferramentas de atividade da doença usadas em práticas clínicas: SLEDAI, SLEDAI-2K ${ }^{39}$, SELENA-SLEDAI, BILAG, Avaliação global do médico e/ou avaliação global do paciente/pai.

7. Utilização de outras ferramentas usadas em práticas clínicas pelos profissionais: SLICC/ACR, SMILEY, CHAQ, CHQ. 
8. Cuidados gerais de suporte disponíveis no local de trabalho (proteção solar, orientação de atividade física, abordagem de equipe multidisciplinar, controle rigoroso da pressão arterial, problemas de função sexual, prescrição de contraceptivos e/ou plano de transição para o médico adulto adequado).

9. Práticas disponíveis para tratamento de pacientes com LESJ internados e ambulatoriais (prontuário eletrônico, hospital-dia, orientação alimentar, prescrição de medicamentos, telefonemas para avaliação clínica, agendamento de consultas, apoio comunitário, cuidados paliativos, atendimento psicológico, atendimento odontológico, fisioterapia, terapia fonoaudiológica, coleta de exames laboratoriais, infiltração intra-articular, administração de terapia intravenosa, aconselhamento a familiares e pacientes sobre a doença, participação em ensaios clínicos, educação de pacientes e familiares sobre a doença, adesão ao tratamento, realização e ensino de atividades físicas, mensuração de sinais vitais, avaliação de dados antropométricos, avaliação de caderneta de vacina, questionários de qualidade de vida relacionados à saúde, avaliação da dor e monitoramento de eventos adversos.

10. Questões observadas em pacientes adolescentes com lúpus (não adesão aos medicamentos, uso de tabaco, ingestão de álcool, uso de drogas ilícitas, câncer e gravidez).

11. Programa centrado na transição para o cuidado de adultos (transferência baseada apenas na idade, transição para um médico de medicina interna, transição para a clínica reumatologica no mesmo serviço de reumatologia, transição para a clínica reumatologica de outro serviço de reumatologia ou programa não estabelecido).

\section{Definições}

- Índice de atividade da doença sistêmica do LES (SLEDAI), instrumento desenvolvido por um grupo de especialistas em Toronto e descrito em detalhes por Bombardier et al. Ele é um indice global que avalia a atividade da doença a partir de 24 itens que incluem manifestações específicas em 9 sistemas orgânicos nos últimos 10 dias com pontuação máxima $105^{38}$ 
- Índice de atividade da doença do lúpus eritematoso sistêmico 2000 (SLEDAI-2K): em 2002 foi lançada uma versão revisada e atualizada do SLEDAl, que incluiu erupção cutânea, alopecia, úlceras ou proteinúria persistente. (Anexo 1) ${ }^{39}$

- Segurança de estrogênios na avaliação nacional do lúpus eritematoso (SELENASLEDAI): nesse instrumento foi adicionado um glossário, além de ter sua pontuação modificada, levando em consideração a persistência da atividade da doença em alguns descritores (alopécia, erupção cutânea, úlceras mucosas) que anteriormente não eram pontuados, a menos que fossem novos ou recorrentes. Nele os pesquisadores pesquisadores aceitaram a presença de achados objetivos ou subjetivos para pontuar o descritor como presente. (Anexo 2$)^{42}$

- British Isles Lupus Assessment Group (BILAG): esse instrumento foi publicado inicialmente em 1988, sendo atualizado em 2004 e publicado em 2005. O índice categoriza a atividade da doença em 5 níveis diferentes de $A$ a $E$ sendo que $A$ representa doença muito ativa e $\mathrm{E}$ nenhuma atividade da doença atual ou anterior. Com ela avaliamos a atividade da doença nas últimas 4 semanas. Ele possui 97 itens que deverão ser respondidos com: 0 = ausente, $1=$ melhorando, $2=$ igual, $3=$ pior ou 4 $=$ novo. $(\text { Anexo } 3)^{42}$

- Índice de danos cumulativo SLICC/ACR (Systemic Lupus Erythematosus Collaborating Clinics/American College of Rhumatology Damage Index): o índice de dano crônico no lúpus eritematoso sistêmico pontua de 0 a 46, e inclui alterações que estejam presentes há pelo menos 6 meses ou que sejam irreversíveis. $O$ índice é cumulativo e valores mais altos estão associados a pior prognóstico e maior mortalidade. $(\text { Anexo } 4)^{38}$

- Medida Simples do Impacto do Lúpus Eritematoso em Jovens (SMILEY): é um questionário de Qualidade de Vida Relacionada a Saúde (QVRS) de 26 itens, específico para LES, que apresenta 4 domínios: efeito sobre o próprio, limitações, social e carga de LES. As respostas são relatadas em uma escala de 5 faces. Cada pontuação varia de 1 a 5 e a pontuação total é transformada em uma escala de 1 a 100 , com valores mais altos representativos de melhor QVRS e confiabilidade interna de 0,9 em outras populações de LES com início na infância. (Anexo 5$)^{43}$ 
- Childhood Health Assessment Questionnaire (CHAQ): é dividido em 30 questões, subdividido em 8 domínios que abrangem os principais aspectos da vida diária: vestir e cuidados pessoais, levantar, comer, caminhar, higiene, alcançar, preensão e atividades. Cada domínio é classificado em uma escala de 4 pontos (sem dificuldade, com alguma dificuldade, com muita dificuldade/ necessita de ajuda e incapaz). $O$ índice de incapacidade é calculado como a média dos 8 domínios, sendo que de cada domínio é considerado a maior pontuação, e sua pontuação varia de: 0 - sem deficiência, 1 - com alguma dificuldade, 2- com muita dificuldade/ necessita de ajuda e 3 - incapaz. A pontuação máxima é 3. (Anexo 6) $)^{44}$

- Child Health Questionnaire (CHQ): é um formulário preenchido pelos pais, possui 50 questões que abrange 10 domínios mentais, físico ou sub escalas: funcionamento físico, dor corporal, percepções gerais de saúde, funções/limitações sociais - físicas, funções /limitações sociais - emocional /comportamental, impacto dos pais -Tempo e o impacto dos pais - emoções, auto-estima, saúde mental e comportamento geral. Cada pontuação de subescala varia de 0 a 100, com pontuações mais altas representando melhor estado de saúde. Duas pontuações, $\mathrm{CHQ}$ - resumo físico e $\mathrm{CHQ}$ - resumo psicossocial, são calculados pela agregação das subescalas (Anexo 7). ${ }^{45}$

\subsection{Análise Estatística}

Os resultados foram apresentados como mediana (variação) ou médiatdesvio padrão para variáveis contínuas e número (\%) para variáveis categóricas. Foram realizadas comparações entre os profissionais quanto ao trabalho em serviço público versus serviço privado, profissionais que cuidaram e não cuidaram de pacientes com LESJ, profissionais que atenderam e não atenderam pacientes hospitalizados, e profissionais que trabalham em serviço público e universidade pública versus os que trabalham em serviço público, todas utilizando as variáveis que possivelmente apresentariam diferenças de atendimento, visando obter diferenças estatisticamente significantes entre os grupos. Os dados foram comparados utilizando-se o teste t e teste de Mann Whitney para as variáveis contínuas. Para variáveis categóricas as diferenças foram avaliadas através do teste do qui-quadrado de Pearson ou teste exato de Fisher. Em todos os testes estatísticos o nível de significância da variável 
independente foi estabelecido em $5 \%(p<0,05)$. As análises foram realizadas no software estatístico SPSS v.18 for Windows.

\subsection{Aspecto ético e legal}

O projeto foi submetido à Comissão de Ética para Análise de Projetos de Pesquisa (CAPPesq) do Hospital das Clínicas de São Paulo e aprovado sob Parecer número 2.089.884. A página inicial do questionário apresentou as informações sobre o consentimento em participar do estudo, conforme previisto na Resolução 466 no Conselho Nacional de Saúde. 
Resultados 


\section{Resultados}

No período de 2018 a 2019 foram enviados mensalmente 373 formulários para enfermeiros dos Estados brasileiros, e em outubro de 2019 foram entregues 30 questionários aos enfermeiros presentes no Congresso Brasileiro da SOBEP que afirmaram terem cuidado de pacientes com LESJ.

Houve retorno de 111 questionários respondidos (29,4\%), provenientes das regiões Sul, Sudeste, Centro-Oeste e Nordeste.

As características sociodemográficas apresentadas na Tabela 1 evidenciaram maioria do sexo feminino $(90,1 \%)$, nacionalidade brasileira $(99,1 \%)$, com idade média de 39,5 anos. Quanto a prática profissional, houve predomínio de trabalho no serviço público (82,9\%), jornada de trabalho em período integral para $46,8 \%$ dos profissionais e quase um terço destes com atuação em unidade de internação (30,6\%). Mais da metade dos enfermeirios não realizou consulta de enfermagem aos seus pacientes $(59,5 \%)$.

Tabela 1: Dados demográficos e caracterização dos enfermeiros

Variáveis $\quad n=111(\%)$

Dados Demográficos

Sexo Feminino

$100(90,1)$

Idade

$39,5(24-61)$

Local de trabalho

Serviço público

$92(82,9)$

Serviço privado

$20(18,0)$

Universidade privada

$19(17,1)$

Universidade pública

$3(2,7)$

Jornada de Trabalho $n=110$

Integral

$52(46,8)$

Meio Período

$34(30,9)$

Outros

$24(21,6)$ 


\begin{tabular}{|c|c|}
\hline Variáveis & $n=111(\%)$ \\
\hline \multicolumn{2}{|c|}{ Atendimento disponível aos pacientes } \\
\hline Serviço Público & $97(87,4)$ \\
\hline Convênio & $35(31,5)$ \\
\hline Filantrópico & $20(18,0)$ \\
\hline Serviço Particular & $18(16,2)$ \\
\hline \multicolumn{2}{|l|}{ Unidade de Trabalho } \\
\hline Unidade Internação & $34(30,6)$ \\
\hline PS & $19(17,1)$ \\
\hline UTI & $15(13,5)$ \\
\hline Unidade cirúrgica & $06(05,4)$ \\
\hline Ambulatório & $05(4,5)$ \\
\hline Hospital Dia & $05(4,5)$ \\
\hline UBS & $(0)$ \\
\hline
\end{tabular}

\section{Consulta de enfermagem}

Não Realizou

$66(59,5)$

Depois do Médico

Antes do Médico

$20(18,0)$

$\mathrm{n}$ apresentado em frequência e porcentagem, $\mathrm{PS}=$ Pronto Socorro, UTI= Unidade de Terapia Intensiva , UBS= Unidade Básica de Saúde

O atendimento dos pacientes caracterizado na Tabela 2 revelou que os principais suportes gerais disponíveis para o tratamento são a abordagem do paciente pela equipe multiprofissional (65,8\%), principalmente de fonoaudiólogos, enfermeiros, nutricionistas e fisioterapeutas, seguido de avaliação da dor (46,8\%). Entre os problemas apresentados no atendimento aos pacientes, $45,1 \%$ dos profissionais identificaram a hospitalização, enquanto 41,4\% não observaram nenhum dos problemas apresentados. Ressalta-se o fato de que 50,5\% desconhecem o processo de transição dos pacientes com LESJ para o serviço adulto. 
Tabela 2: Caracterização do atendimento dos profissionais

\begin{tabular}{lcc}
\hline Variáveis & $\mathbf{n}=\mathbf{1 1 1}(\%)$ \\
\hline Cuidados de suporte gerais disponíveis & $73(65,8)$ \\
Abordagem pela equipe multidisciplinar & $52(46,8)$ \\
Controle da pressão arterial & $43(38,7)$ \\
Protetor Solar & $18(16,2)$ \\
Orientação para atividade física & $14(12,6)$ \\
Plano de transição para um profissional de saúde do adulto & $07 \quad(6,3)$ \\
Orientação da função sexual & $05 \quad(4,5)$ \\
Uso de Contraceptivos & & \\
\hline Profissionais com quem trabalhou & $106 \quad(95,5)$ \\
Fonoaudiologo & $96(86,5)$ \\
Enfermeiro & $93(83,8)$ \\
Nutricionista & $93(83,8)$ \\
Fisioterapeuta & $78(70,3)$ \\
Educador Físico & $65(58,6)$ \\
Assistente Social & $54(48,6)$ \\
Biomédico & $43(38,7)$ \\
Médico & $41 \quad(36,9)$ \\
Farmacêutico & $26(23,4)$ \\
Psicologo & $18(16,2)$ \\
Dentista & $05 \quad(4,5)$ \\
Terapeuta Ocupacional & $02 \quad(1,8)$ \\
\hline Problemas observados com os pacientes & $51 \quad(45,9)$ \\
Hospitalização & $46 \quad(41,4)$ \\
Não observei nenhum desses problemas & $31 \quad(27,9)$ \\
Má aderência medicamentosa & $06 \quad(0,4)$ \\
Gravidez & $03 \quad(2,7)$ \\
Consumo de álcool & $03 \quad(2,7)$ \\
Uso de drogas ilícitas & $03 \quad(2,7)$ \\
Câncer & 02 \\
Consumo de cigarro &
\end{tabular}




\begin{tabular}{lcc}
\hline Variáveis & $\mathbf{n}=\mathbf{1 1 1}(\%)$ \\
\hline Estratégia de transição para cuidado adulto & $56 \quad(50,5)$ \\
Desconheço o processo de transição de meu serviço & $17 \quad(15,3)$ \\
Transição para reumatologista adulto no mesmo serviço & $17 \quad(15,3)$ \\
Não há programa estabelecido & $11 \quad(9,9)$ \\
Transferência baseada somente na idade & $09 \quad(8,1)$ \\
Transição clínica para adultos no mesmo serviço & $09 \quad(8,1)$ \\
Transição para reumatologista adulto em outro serviço & &
\end{tabular}

Quanto ao conhecimento da doença e atendimento de pacientes, observou-se que 51 profissionais que responderam a essa pergunta, cuidaram de pacientes com LESJ (45,9\%). A avaliação do LESJ, descrita na Tabela 3, revela que mais da metade dos profissionais não utiliza nenhuma ferramenta para avaliar a atividade do LESJ nos pacientes e mais de um terço desconhece a existência destas ferramentas:

Tabela 3: Conhecimento dos enfermeiros sobre as ferramentas para avaliar o LESJ

\begin{tabular}{|c|c|}
\hline Variáveis & $n=111(\%)$ \\
\hline \multicolumn{2}{|c|}{ Ferramentas utilizadas para avaliar atividade da doença } \\
\hline \multicolumn{2}{|l|}{ Nenhuma } \\
\hline \multirow{2}{*}{ Eu não conheço essas ferramentas } & $57(51,4)$ \\
\hline & $42(37,8)$ \\
\hline SLEDAI & $19(17,1)$ \\
\hline SLEDAI 2K & $19(18,0)$ \\
\hline Avaliação Global pelo paciente/pais & $04 \quad(3,6)$ \\
\hline SELENA & \\
\hline \multirow[t]{2}{*}{ BILAG } & (U) \\
\hline & (0) \\
\hline
\end{tabular}




\begin{tabular}{|c|c|c|}
\hline Variáveis & $\mathbf{n}=$ & $11(\%)$ \\
\hline \multicolumn{3}{|c|}{ Outras ferramentas utilizadas na prática clínica } \\
\hline Nenhuma & 55 & $(49,5)$ \\
\hline Eu não conheço essas ferramentas & 50 & $(45,0)$ \\
\hline SLICC/ACR-DI & 15 & $(13,5)$ \\
\hline CHAQ & 01 & $(9,0)$ \\
\hline SMILEY & 0 & (0) \\
\hline $\mathrm{CHQ}$ & 0 & \\
\hline \multicolumn{3}{|c|}{$\begin{array}{l}\text { n apresentado em frequência e porcentagem. SLEDAI = Índice de atividade da doença } \\
\text { sistêmica do LES, SLEDAI } 2 \mathrm{~K}=\text { Índice de atividade da doença do lúpus eritematoso } \\
\text { sistêmico 2000, SELENA = Segurança de estrogênios na avaliação nacional do lúpus } \\
\text { eritematoso, BILAG = British Isles Lupus Assessment Group, SLICC/ACR-DI Índice de danos } \\
\text { cumulativo pelo Systemic Lupus Erythematosus Collaborating Clinics/American College of } \\
\text { Rhumatology Damage Index, CHAQ = Childhood Health Assessment Questionnaire, } \\
\text { SMILEY = Simple Measure of the Impact of Lupus Erythematosus in Younger, CHQ = Child } \\
\text { Health Questionnaire }\end{array}$} \\
\hline
\end{tabular}

A Tabela 4 descreve os procedimentos e terapêutica disponíveis aos pacientes com LESJ realizados pelos enfermeiros:

Tabela 4: Procedimentos e Terapêutica disponíveis aos pacientes com LESJ

\begin{tabular}{lll}
\hline Variáveis & $\mathbf{n = 1 1 1}(\%)$ \\
\hline Avaliação da dor & $52 \quad(46,8)$ \\
Coleta de exames laboratoriais & $50 \quad(45,0)$ \\
Mensuração de sinais vitais & $49 \quad(44,1)$ \\
Administração de terapia intravenosa & $45 \quad(40,5)$ \\
Prontuário Eletrônico & $42 \quad(37,8)$ \\
Monitoramento de eventos adversos & $32 \quad(28,8)$ \\
Informação do paciente para outros & $31 \quad(27,9)$ \\
profissionais/departamentos &
\end{tabular}




\begin{tabular}{lll}
\hline Variáveis & $\mathbf{n}=\mathbf{1 1 1}(\%)$ \\
\hline Prescrição Medicamentosa & $30 \quad(27,0)$ \\
Educação do paciente/família sobre a doença & $26 \quad(23,4)$ \\
Aconselhamento do paciente/família sobre a doença & $24 \quad(21,6)$ \\
Avaliação da caderneta de vacinação & $20 \quad(18,0)$ \\
Verificação de aderência medicamentosa & $17 \quad(15,3)$ \\
Agendamento de consultas & $16 \quad(14,4)$ \\
Hospital Dia & $15 \quad(13,5)$ \\
Avaliação de dados antropométricos & $15 \quad(13,5)$ \\
Orientação Nutricional & $13 \quad(11,7)$ \\
Cuidado Paliativo & 11 & $(9,9)$ \\
Aderência às consultas & 09 & $(8,1)$ \\
Questionários relacionados a qualidade de vida & 09 & $(8,1)$ \\
Reabilitação & 08 & $(7,2)$ \\
Fonoaudiologia & 08 & $(7,2)$ \\
Administração de injeção intrarticular & $08 \quad(7,2)$ \\
Participação em estudos clínicos & $08 \quad(7,2)$ \\
Fisioterapia & $07 \quad(6,3)$ \\
Tratamento Odontológico & $06 \quad(5,4)$ \\
Realizar/ensinar atividades físicas & $05 \quad(4,5)$ \\
Chamadas telefônicas para lembrar suas consultas & $03 \quad(2,7)$ \\
Auxiliar para os recursos da comunidade & $03 \quad(2,7)$ \\
\hline napresen & &
\end{tabular}

$\mathrm{n}$ apresentado em frequência e porcentagem.

Em relação ao que é necessário para realizar uma melhor prática ao paciente com LESJ, observa-se, na Tabela 5, a necessidade de conhecer o tratamento da doença (83,8\%), realizar um cuidado que contemple o paciente e a família $(64, \%)$, e motivar a aderência ao tratamento aos pacientes $(61,3 \%)$, como principais aspectos revelados. 
Tabela 5: Práticas para melhor assistência aos pacientes com LESJ

\begin{tabular}{lc}
\hline Variáveis & $\mathbf{n = 1 1 1}(\%)$ \\
\hline Práticas para melhor assistência aos pacientes com LESJ & \\
Conhecimento do tratamento & $\mathbf{9 3}(\mathbf{8 3 , 8 )}$ \\
Cuidado ao paciente e à família & $\mathbf{7 1}(64,0)$ \\
Aderência ao tratamento & $\mathbf{6 8}(61,3)$ \\
Habilidade em trabalhar com equipe multiprofissional & $65(58,6)$ \\
Boa comunicação & $64(57,7)$ \\
Abordagem familiar & $60(54,1)$ \\
Avaliação do paciente e cuidado clínico & $57(51,4)$ \\
Experiência em reumatologia & $25(22,5)$ \\
Recursos financeiros & $24(21,6)$ \\
Outra(s): & $3(2,7)$ \\
\hline
\end{tabular}

$\mathrm{n}$ apresentado em frequência e porcentagem

Foram realizadas comparações adicionais entre os profissionais quanto ao trabalho em serviço público versus serviço privado, profissionais que cuidaram e não cuidaram de pacientes com LESJ, profissionais que atenderam e não atenderam pacientes hospitalizados, e profissionais que trabalham em serviço público e universidade pública versus os que trabalham em serviço público, todas utilizando variáveis possiveis de apresentar diferenças de atendimento: cuidado ao paciente com LESJ, paciente hospitalizado, consulta de enfermagem, avaliação de caderneta de vacinação, cuidado paliativo, trabalho em equipe multiprofissional, aderência medicamentosa, uso de protetor solar, utilização e conhecimento de ferramentas de mensuração da atividade da doença.

A análise dos profissionais que trabalham apenas no serviço público versus aqueles que atuam apenas no serviço privado não evidenciou nenhuma diferença de significância estatística quanto ao atendimento de pacientes hospitalizados, inclusive quanto à orientação de protetor solar, aplicação de questionário de qualidade de vida, avaliação de caderneta de vacinação e utilização de cuidado paliativo, todos relevantes aos pacientes com LESJ. 
Tabela 6: Atendimento exclusivo do serviço público versus serviço privado

\begin{tabular}{lccc}
\hline Variáveis & Serviço & Serviço & \\
& $\begin{array}{c}\text { Público } \\
\text { N=69 (\%) }\end{array}$ & Privado & N=8 (\%) \\
\hline Cuidou de Pacientes com LESJ & $39(56,5)$ & $2(25,0)$ & 0,137 \\
Hospitalização & $33(47,8)$ & $2(25,0)$ & 0,279 \\
Não fez consulta de enfermagem & $63(91,3)$ & $8(100)$ & 1,000 \\
Avaliação caderneta vacinação & $14(20,2)$ & $1(12,5)$ & 1,000 \\
Orientou uso de Protetor Solar & $33(47,8)$ & $1(12,5)$ & 0,071 \\
Verificou má aderência medicamentosa & $20(28,9)$ & $2(25,0)$ & 1,000 \\
Realizou Cuidado Paliativo & $06(8,6)$ & $1(12,5)$ & 0,551 \\
Trabalho com equipe multiprofissional & $45(65,2)$ & $4(50,0)$ & 0,452 \\
Questionário de qualidade de vida & $5(7,2)$ & $0(0,0)$ & 1,000 \\
\hline Ferramentas de atividade da doença & & & \\
Não conhece & $26(37,6)$ & $3(37,5)$ & 1,000 \\
Não utiliza nenhuma & $32(46,3)$ & $5(62,5)$ & 0,722 \\
\hline Outras ferramentas para utilizadas & & & \\
Não conhece & $30(43,4)$ & $4(50,0)$ & 0,726 \\
Não utiliza & $33(47,8)$ & $4(50,0)$ & 1,000 \\
\hline
\end{tabular}

$\mathrm{n}$ apresentado em frequência e porcentagem

A análise feita entre profissionais que já cuidaram de pacientes com LESJ no último ano, independente do tipo de serviço, e os que não cuidaram revelou difereças estatisticamente significantes apenas na avaliação de caderneta de vacinação e tipo de trabalho, conforme mostra a Tabela 7: 
Tabela 7: Análise entre profisisonais que já cuidaram de pacientes com LESJ

\begin{tabular}{|c|c|c|c|}
\hline Variáveis & $\begin{array}{l}\text { Já cuidou de } \\
\text { pacientes } \\
\text { com LESJ } \\
\mathrm{N}=62(\%)\end{array}$ & $\begin{array}{l}\text { Não cuidou } \\
\text { de pacientes } \\
\text { com LESJ } \\
\mathrm{N}=19(\%)\end{array}$ & $\mathbf{P}$ \\
\hline \multicolumn{4}{|l|}{ Trabalho no serviço privado } \\
\hline Trabalho no serviço público & $9(14,5)$ & $7(36,8)$ & $<0,05$ \\
\hline Não realizou consulta de & $16(25,8)$ & $48(77,4)$ & 0,749 \\
\hline enfermagem & $56(90,3)$ & $18(94,7)$ & 1,000 \\
\hline Avaliação caderneta vacinação & $8(12,9)$ & $7(36,8)$ & $<0,05$ \\
\hline Realizou Cuidado Paliativo & $5(8,1)$ & $3(15,8)$ & 0,383 \\
\hline Orientou uso de protetor solar & $23(37,1)$ & $4(21,1)$ & 0,269 \\
\hline Trabalho com Equipe & $46(74,2)$ & $10(52,6)$ & 0,093 \\
\hline multiprofissional & $4(6,5)$ & $2(10,5)$ & 0,621 \\
\hline
\end{tabular}

Ferramentas de atividade da doença

Nenhuma $32(51,6) \quad 9(47,4) \quad 0,798$

Eu não conheço essas $28(45,2) \quad 12(63,2)$ 0,198 ferramentas

\section{Outras ferramentas para}

utilizadas

Nenhuma

Eu não conheço essas $29(46,8)$

$8(42,1)$

0,796

ferramentas

$36(58,1) \quad 14(73,7)$

0,285

$\mathrm{n}$ apresentado em frequência e porcentagem

A Tabela 8 comparou os profissionais quanto ao atendimento ou não de pacientes com LESJ hospitalizados, e revelou que o cuidado paliativo é mais realizado de maneira significante por enfermeiros que não atenderam paciente hospitalizado $(p<0.0001)$, enquanto que a orientação de protetor solar $(p<0.05)$ e trabalho em equipe 
multiprofissional $(p<0.0001)$, são significantemente mais realizados por aqueles que atenderam pacientes internados .

Tabela 8: Análise entre profissionais que atenderam pacientes com LESJ hospitalizados

\begin{tabular}{lrcc}
\hline Variáveis & $\begin{array}{c}\text { Paciente com } \\
\text { LESJ }\end{array}$ & $\begin{array}{c}\text { Sem paciente } \\
\text { com LESJ }\end{array}$ & P \\
& $\begin{array}{c}\text { hospitalizado } \\
\text { N=51(\%) }\end{array}$ & $\begin{array}{c}\text { hospitalizado } \\
\mathbf{N}=60(\%)\end{array}$ & \\
\hline Trabalho no serviço privado & & & \\
Trabalho no serviço público & $2(3,9)$ & $6(10,0)$ & 0,284 \\
Não realizou consulta de & $33(64,7)$ & $36(60,0)$ & 0,696 \\
enfermagem & $45(88,2)$ & $56(93,3)$ & 0,508 \\
Avaliação caderneta vacinação & $12(23,5)$ & $8(13,3)$ & 0,217 \\
Realizou Cuidado Paliativo & $6(11,7)$ & $37(61,6)$ & $<\mathbf{0 , 0 0 0 1}$ \\
Orientou uso de protetor solar & $28(54,9)$ & $15(25,0)$ & $<0,05$ \\
Trabalho com Equipe & $43(84,3)$ & $30(50,0)$ & $<0,0001$ \\
multiprofissional & $4(7,8)$ & $5(8,3)$ & 1,000 \\
Questionário qualidade de vida & & & \\
\hline Ferramentas de atividade da & & & 0,255 \\
doença & $22(43,1)$ & $33(55,0)$ & 0,434 \\
Nenhuma & $22(43,1)$ & $35(58,3)$ & 0,129 \\
Eu não conheço essas ferramentas & $20(39,2)$ & $22(36,6)$ & 0,846 \\
\hline Outras ferramentas para & & & \\
utilizadas & & & \\
Nenhuma & & & \\
Eu não conheço essas ferramentas & & & \\
\hline & & & \\
\hline
\end{tabular}

$\mathrm{n}$ apresentado em frequência e porcentagem.

A última comparação, feita entre profissionais que trabalham em serviço público e universidade públlica versus os que só trabalham em serviço público, evidenciou diferença apenas na aplicação de questionário de qualidade de vida, conforme ilustra a Tabela 9: 
Tabela 9: Profissionais que trabalham em serviço e universidade pública $\mathrm{x}$ serviço público

\begin{tabular}{|c|c|c|c|}
\hline Variáveis & $\begin{array}{l}\text { Serviço e } \\
\text { Univ pública } \\
\text { N=9(\%) }\end{array}$ & $\begin{array}{l}\text { Serviço } \\
\text { público } \\
\text { N=72 (\%) }\end{array}$ & $\mathbf{P}$ \\
\hline $\begin{array}{l}\text { Não realizou consulta de } \\
\text { enfermagem }\end{array}$ & $8(88,8)$ & $66(91,6)$ & 0,576 \\
\hline Avaliação caderneta vacinação & $2(22,2)$ & $15(20,8)$ & 1,000 \\
\hline Realizou Cuidado Paliativo & $2(22,2)$ & $6(8,3)$ & 0,216 \\
\hline Orientou uso de protetor solar & $5(55,5)$ & $34(47,2)$ & 0,732 \\
\hline Trabalho com Equipe & $7(77,7)$ & $47(65,2)$ & 0,710 \\
\hline \multicolumn{4}{|l|}{ Multiprofissional } \\
\hline Questionário qualidade de vida & $3(33,3)$ & $5(6,9)$ & $<0,05$ \\
\hline \multicolumn{4}{|l|}{$\begin{array}{l}\text { Ferramentas de atividade da } \\
\text { doença }\end{array}$} \\
\hline Nenhuma & $2(22,2)$ & $33(45,8)$ & 0,287 \\
\hline Eu não conheço essas ferramentas & $5(55,5)$ & $29(40,2)$ & 0,481 \\
\hline \multicolumn{4}{|l|}{$\begin{array}{l}\text { Outras ferramentas para } \\
\text { utilizadas }\end{array}$} \\
\hline Nenhuma & $6(66,6)$ & $34(47,2)$ & 0,312 \\
\hline Eu não conheço essas ferramentas & $2(22,2)$ & $33(45,8)$ & 0,287 \\
\hline
\end{tabular}

n apresentado em frequência e porcentagem. 
Discussão 


\section{Discussão}

Este é o primeiro trabalho em nível nacional que buscou descrever o atendimento dos enfermeiros no tratamento do LESJ, através de um questionário autoaplicável.

A complexidade desta doença autoimune que acomete vários sistemas, causa dores e pleurisia no paciente, ocasiona incapacidade física e por vezes dependência física. Principalmente no início da doença, impossibilita ao paciente estudar, trabalhar e realizar atividades de lazer, além de alterar a imagem corporal quando existe acometimento cutâneo ${ }^{45-46}$. Ademais, existe sensação de ansiedade, distúrbio de humor e depressão ${ }^{46-47}$, um tratamento com diversos medicamentos que ocasionam efeitos colaterais e sintomatologia algumas vezes mais severas que a própria doença ${ }^{42}$.

Este quadro complexo demanda um atendimento multiprofissional, que contemple todas as esferas acometidas, e este estudo evidenciou tal necessidade por parte dos enfermeiros $(65,8 \%)$, ratificando a necessidade do atendimento multiprofissional com enfoque na aderência do paciente e família ao tratamento. Um estudo de desenho semelhante realizado recentemente com médicos reumatologistas atuantes na América Latina também identificou a importância da equipe multiprofissional no tratamento dos pacientes e famílias, visando aderência e controle da atividade da doença ${ }^{36}$.

Como mencionado, a aderêcia é um ponto relevante do tratamento, e este estudo também verificou uma preocupação por parte dos profissionais $(61,3 \%)$ quanto a esta temática. Corroborando com este fato, um estudo realizado na China, liderado por um farmacêutico, avaliou o atendimento multidisciplinar e evidenciou melhora na remissão da doença e concomitante melhora na qualidade de vida e aderência do grupo intervenção, devido ao fato de que o atendimento foi centrado no paciente. Os profissionais realizavam reuniões regulares e quando o paciente não ia ao serviço, era realizado contato telefônico, para garantir que recebesse orientações de saúde. ${ }^{42}$

Quando o tratamento envolve o paciente adolescente, devem ser considerados outros fatores inerentes a este período que compreende a maturação estrutural e funcional do cérebro, como presença de comorbidades, dor, uso contínuo de medicação, consultas médicas e com outros profissionais, sequelas e atividade da doença, que pode trazer mudanças na imagem corporal, nas responsabilidades de auto-cuidado e culminar em isolamento social. Também podem ocorrer as questões da 
própria adolescência, como uso de álcool e tabaco, dúvidas quanto a identidade sexual, gravidez, violência, bullying, o que exige um preparo dos profissionais e conhecimento da fisiopatologia do LESJ para atingir todos os níveis necessários de intervenção.

Este despreparo ou lacuna de conhecimento sobre o LESJ na população estudada, evidenciado em $83,8 \%$ dos enfermeiros que relataram a necessidade de conhecer o tratamento para uma melhor assistência ao paciente lúpico, também foi identificado na literatura sobre os cuidados de enfermagem relacionados ao portador de LESJ. Um relato de experiência publicado para citar os cuidados de enfermagem a esses pacientes, encontrou apenas dois artigos que apresentavam cuidados de enfermagem ${ }^{50}$, o que permite inferir que tal necessidade de capacitação não se resume ao tratamento, fisiopatologia, sinais e sintomas da doença, mas também de melhorar a assistência a esses pacientes.

E, embora exista esse desconhecimento sobre a complexidade da doença, não se pode afirmar que esta seja a razão dos números baixos de respostas referentes aos cuidados de LESJ realizados, como cuidado paliativo, questionário de qualidade de vida, aderência medicamentosa e de tratamento, orientação para prática de atividade física, que são esperadas para qualquer paciente com doença crônica. As comparações realizadas entre os profissionais mostraram que estes cuidados direcionados não ocorreram independente do tipo de vínculo, atendimento e cuidado realizado, corroborando para a importância da disseminação do conhecimento nas instituições em geral, considerando a complexidade da doença, número pequeno de especialistas, dificuldade de diagnóstico precoce e direcionamento de tratamento aos serviços de referência.

Atualmente existem vários instrumentos para medir ou avaliar a qualidade de vida, que incuem domínios Um aspecto intrínseco e relevante ao paciente com LESJ diz respeito a transição para o cuidado adulto. A formação de grupos de estudos sobre problemas relacionados a transição de cuidados que ocorreu em 2010 culminou na elaboração de recomendações, programas, ferramentas, com intuito de propiciar opções aos profissionais de saúde, adolescentes e seus familiares a enfrentarem juntos este processo ${ }^{45-48}$. Tais programas visam preparar o adolescente 0 mais precocemente possível para o desenvolvimento de independência e para 0 auto cuidado (a partir dos 12 anos), acompanhando o processo de transição, avaliação de prontidão de transição, planejamento para a transição por meio de identificação das 
prioridades dos adolescentes especialmente para tomadas de decisões a partir dos 16 anos, transferência e integração aos cuidados do adulto e conclusão da transferência.

Com essas premissas estabelecidas, foi realizado um trabalho em um serviço público materno-infantil terciário do Rio de Janeiro, que buscou verificar as condições de transição implementadas no seus serviços de especialidades pediátricas. Neste estudo os autores observaram que de 5 unidades avaliadas, apenas três possuem um ou mais profissionais dedicados ao processo de transição, mesmo que não sejam exclusivos, que em nenhuma das unidades ocorre consulta conjunta entre o pediatra e o médico de adulto, e nem utilizam ferramentas validadas para auxiliar a avaliação das habilidades do adolescente ao longo do processo ou conta com programas educativos específicos para a transiçãa ${ }^{55}$.

Outro estudo realizado no Brasil, avaliou as práticas de transição em centros de reumatologia pediátrica no Brasil, utilizando um questionário modificado previamente aplicado a reumatologistas pediátricos nos EUA e Canadá, nesse estudo observaram que as políticas dão poucas e não padronizadas, podendo levar a mau prognósticos e resultados insatisfatórios ${ }^{56}$. Os resultado do presente estudo revelaram que $50,5 \%$ dos enfermeiros desconhecem o processo institucional de transição do paciente com LESJ do serviço pediátrico para o serviço adulto, fazendo-se necessária a criação de protocolos e/ou melhor disseminação de protocolos existentes nas instituições, para orientar o profissional a participar deste processo a fim de preparar os pacientes com doenças crônicas como LESJ e suas famílias, para uma nova realidade: ser um adulto com doença crônica.

A relevância deste trabalho justifica-se pela escassez de pesquisas com informações sobre $o$ atendimento do enfermeiro ao paciente com LESJ. Os trabalhos existentes sobre o tema, abordam a doença, quanto a atividade, o prognóstico e complicações associadas, mas não mostram a importância do diagnóstico precoce ou do reconhecimento de atividade da doença, do conhecimento dos próprios pacientes e familiares sobre a doença, ou da utilização de intrumentos que auxiliam a verificar a adesão, qualidade de vida, aceitação da própria doença.

Os profissionais participantes realizam os cuidados de rotina aos pacientes mas não utilizam ferramentas para avaliar a atividade da doença nem ferramentas auxiliares ao tratamento, além de atividades esperadas aos pacientes com LESJ, como questões relacionadas à aderência e transição ao cuidado adulto, que podem estar relacionadas à lacuna de conhecimento. 
A consulta de enfermagem, já mencionada, constitui-se em atividade exclusiva do enfermeiro, é relevante e permite identificar problemas de aderência, de relacionamento familiar, de conhecimento e de aceitação da doença. Sua eficácia depende diretamente do conhecimento do enfermeiro, mas não deve limitar-se a isto, principalmente nos casos de primeiro atendimento. Entendemos que nesses casos o ideal seja realizar uma consulta diagnóstica e então buscar respostas mediante os achados mais importantes que precisam de solução.

É possível verificar a aderência ao tratamento medicamentoso utilizando instrumentos disponíveis na prática clínica, como o Teste de Morisky e Green (TMG) ${ }^{57}$, composto de quatro perguntas que identificam atitudes e comportamentos dos pacientes quanto a ingesta de remédios, tem rápida aplicação e permite um diagnóstico simples. Este instrumento é de grande valia nos casos de atividade da doença e consequente necessidade de vários medicamentos.

Para desempenhar com qualidade a competência de educador, o enfermeiro deve buscar aprimoramento contínuo na área de atuação, e tratando-se do cuidado ao paciente com LESJ a orientação de proteção solar é de suma importância. Além do fato de que estudos demonstram que $50 \%$ da exposição ao longo da vida aos raios ultravioletas (RUV) ocorrem até os 18 anos de idade, a fotossensibilidade ocasionada pela exposição aos RUV, pode manifestar-se não somente com erupções cutâneas ou fadiga, como também artralgia e risco de exacerbação da doença em órgãos-alvo, como na nefrite lúpica ${ }^{58-59}$.

O cuidado paliativo é outro aspecto que deve ser considerado, muitas vezes sendo necessário iniciar pela desmistificação deste cuidado, visto frequentemente como um cuidado a ser prestado apenas aos pacientes fora de possibilidade terapêutica por profissionais de saúde que prestam cuidado direto, inclusive.

A Organização Mundial de Saúde ${ }^{60}$ define os cuidados paliativos como uma abordagem que melhora a qualidade de vida dos pacientes (adultos e crianças) e de suas famílias/cuidadores que enfrentam problemas associados a doenças potencialmente fatais, sejam eles físicos, psicológicos, sociais e espirituais. Previne e alivia o sofrimento por meio da identificação precoce, avaliação correta e tratamento da dor e outros problemas, sejam físicos, psicossociais ou espirituais.

Nesse sentido, o ideal é iniciar o cuidado paliativo ao paciente com doença crônica após o diagnóstico, independente do estágio da doença. Também é importante que a falta de uma equipe multiprofissional ou grupo de cuidados paliativos institucional 
não sejam considerados barreiras para a realização deste tipo de cuidado, uma vez que sua execução majoritariamente independe de recursos materiais ou financeiros, e o enfermeiro pode, além de realizá-lo, orientar a equipe de enfermagem e a equipe multiprofissional sobre a realização embasado em evidências publicadas.

A dor, sentimento limitante e incapacitante, faz parte da rotina de muitos pacientes com LESJ em atividade da doença, associada a artrite, úlceras orais ou nasais, infecção. A avaliação da dor e analgesia se necessário também estão entre as atividades do enfermeiro, que precisa utilizar a escala adequada a idade e nível de compreensão do paciente, inclusive daqueles que estão hospitalizados e por vezes sem condições de verbalizar a dor.

A vacinação do paciente lúpico deve estar sempre atualizada, e a verificação da caderneta de vacina deve fazer parte da consulta, pois existe maior risco de infecç̧ão devido ao acometimento imunológico da doença, em especial hespes zoster para aqueles que tiveram varicela prévia, e despende de atenção especial, principalmente se o paciente está em uso de imunossupressores, corroborando a importância do conhecimento profissional sobre a doença.

A Sociedade Brasileira de Reumatologia orienta que vacinas com agentes vivos atenuados são contraindicadas durantes os estados de alta imunossupressão. É recomendado que o paciente receba a vacina com vírus inativado entre duas a quatro semanas antes de iniciar o tratamento e, caso não seja possível esperar para iniciar o tratamento, elas podem ser administradas posteriormente, mas o efeito será reduzido. Se durante o tratamento for necessário a administração de vacina com vírus atenuado, o tratamento deverá ser interrompido de 5 dias até 6 meses, dependendo do imunossupressor utilizado. ${ }^{61}$

Atender o paciente separado do familiar em algum momento de todas as consultas compreende outra atividade de grande importância para os pacientes com LESJ, pois pode-se identificar alguns problemas relacionados a sexualidade, uso de drogas licitas ou ilicitas, violência física, ideação suicida, bulliyng, e o enfermeiro, em poder de tais informações, direciona melhor as intervenções e aciona outros profissionais da equipe mediante os achados.

$O$ fato de o LESJ acometer mais o sexo feminino também merece atenção do enfermeiro, principalmente quando a paciente está na adolescência e tem risco de engravidar. Em 2018 a OMS divulgou relatório com estimativa da existência anual de 12,8 milhões de nascimentos entre meninas adolescentes de 15 a 19 anos $^{62}$. Há 
relatos que o comportamento sexual de adolescentes com doenças crônicas não são diferentes das adolescentes sem comorbidades. Um estudo realizado na California, demonstrou que as adolescentes e as jovens adultas apresentam um maior risco para parto prematuro, aborto espontâneo ou induzido, pré-eclampsia e eclampsia, morte materna e fetal. ${ }^{63}$ Devido a isso a adolescente grávida com lupus demanda um acompanhamento direcionado, que envolve possível necessidade de mudança de medicação e olhar acurado se estiver com a doença ativa. Ainda não existem muitos estudos que orientem os profissionais quanto a utilização de medicamentos para essa faixa etária. A Aliança Europeia de Associações de Reumatologia (EULAR) publicou uma orientação sobre os medicamentos que devem ser evitados durante a gestação de pacientes adultos: micofenolato mofetil, ciclofosfamida, metotrexato, inibidores da ECA, captopril, enalapril, bloqueadores do receptor da angiotensina II, clorotiazida, metropolol e atenolol, bifosfanatos, derivados cumarínicos, anticoagulantes orais, persatin, clopidogrel, prasugrel. ${ }^{64}$

As limitações deste estudo compreendem o baixo retorno de respostas por parte dos profissionais, mesmo com envio programado por um período considerável dos questionários, assim como verificado em outros trabalhos de desenho semelhante, em ressarcimento pelos questionários respondidos, e respostas incompletas, além do pequeno número de profissionais indicados pelos médicos reumatologistas pediátricos brasileiros, que por sua vez são poucos se considerarmos o tamanho do Brasil e o acesso distinto aos serviços de saúde nas diferentes regiões existentes.

Outro ponto que deve ser discutido diz respeito a formação do profissional enfermeiro, não sendo possivel afirmar que doenças crônicas raras e complexas como LESJ façam parte da grade curricular de todas as instituições de ensino, fato que somado a dificuldade do diagnóstico precoce e falta recursos diagnósticos em muitos hospitais ou unidades básicas, corrobora a necessidade de disseminação da informação e capacitação para o melhor tratamento. 
Conclusão 


\section{Conclusões}

- A frequencia de atendimento de crianças e adolescentes com LESJ por enfermeiros brasileiros foi de 45,9\%.

- $51,4 \%$ dos profissionais não utiliza ferramentas durante atendimento aos pacientes com LESJ;

- Não houve diferença de significância estatística quanto ao atendimento de pacientes hospitalizados, inclusive quanto à orientação de protetor solar, aplicação de questionário de qualidade de vida, avaliação de caderneta de vacinação e utilização de cuidado paliativo, todos relevantes aos pacientes com LESJ entre profissionais que trabalham no serviço público e os que trabalham no serviço privado;

- Profissionais que não cuidaram de pacientes com LESJ trabalham significantemente mais no serviço público ( $14.5 \%$ vs $36.8 \%, p<0.05)$ e verificaram mais caderneta de vacinação $(12.9 \%$ vs $36.8 \%, p<0.05)$ do que aqueles que cuidaram de pacientes com LESJ.

- O cuidado paliativo é mais realizado de maneira significante por enfermeiros que não atenderam paciente hospitalizado (11.7\% vs 61.6\%, p<0.0001), enquanto que a orientação de protetor solar $(54.9 \%$ vs $25 \%$, p<0.05) e trabalho em equipe multiprofissional ( $84.3 \%$ vs $50 \%, p<0.0001)$, são significantemente mais realizados por aqueles que atenderam pacientes internados.

- O desconhecimento da doença foi um fator comum e de maior frequência entre os profissionais;

- Existe a necessidade de maior conhecimento sobre o LESJ, sobre o tratamento e formas de melhorar a aderência.

\section{Considerações Finais}

Mediante os achados deste estudo, pretende-se elaborar um aplicativo com o objetivo de otimizar o atendimento do enfermeiro ao paciente com LESJ, dividido em seções que auxiliem no levantamento de sinais e sintomas clínicos, na rotina do paciente, no tratamento medicamentoso, na qualidade de vida, no relacionamento familiar, no status da doença e comorbidades, na necessidade de iniciar ou não um trabalho de transição. 
Anexos 


\section{Anexos}

Anexo 01: Questionário de avaliação do atendimento dos enfermeiros aos pacientes com Lupus Eritematosos Sistêmico Juvenil

Confidential

Questionário para enfermeiros

Please complete the survey below.

Thank you!

Idade em anos:

\begin{tabular}{|c|c|}
\hline Nacionalidade: & $\begin{array}{l}\bigcirc \text { Brasil } \\
\text { Argentina } \\
\text { Bolívia } \\
\text { Chile } \\
\text { Colômbia } \\
\bigcirc \text { Costa Rica } \\
\text { Cuba } \\
\text { Equador } \\
\text { El Salvador } \\
\text { Guatemala } \\
\text { Gaiti } \\
\text { Honduras } \\
\text { México } \\
\text { Nicarágua } \\
\text { Panamá } \\
\text { Paraguai } \\
\text { Peru } \\
\text { República Dominicana } \\
\text { Uruguai } \\
\text { Venezuela } \\
\text { Outra }\end{array}$ \\
\hline
\end{tabular}

Outra:

Sexo: $\quad \bigcirc$ Feminino

Onde você trabalhou no último ano? (Você pode $\quad \square$ Universidade pública

marcar mais de uma resposta)

Serviço público

$\square$ Serviço privado

Qual setor você trabalha? Você pode marcar mais de uma resposta.

$\square$ Pronto Socorro

$\square$ Terapia Intensiva

$\square$ Unidade de internação

$\square$ Unidade cirúrgica

Ambulatório

Hospital dia

$\square$ UBS/Saúde pública

Qual foi a jornada de trabalho que você dedicou aos $\quad \bigcirc$ Meio período (mais de um vínculo empregatício) pacientes no último ano?

Tempo Integral (só um vínculo empregatício)

Outra:

Outra: 


\section{Confidential}

\begin{tabular}{|c|c|}
\hline $\begin{array}{l}\text { Quais os atendimentos disponíveis para seus pacientes } \\
\text { acompanhados no último ano? Você pode marcar mais de } \\
\text { uma resposta. }\end{array}$ & $\begin{array}{l}\square \text { Serviço Público } \\
\square \text { Serviço Particular } \\
\square \text { Convênio } \\
\square \text { Filantrópico }\end{array}$ \\
\hline $\begin{array}{l}\text { Você realizou consulta antes ou após o atendimento } \\
\text { médico no último ano? }\end{array}$ & $\begin{array}{l}\text { Antes do médico } \\
\text { Após o médico } \\
\text { Não, eu não realizei consulta }\end{array}$ \\
\hline $\begin{array}{l}\text { Seus pacientes participaram de algum estudo clínico } \\
\text { no último ano? }\end{array}$ & $\underset{\text { Sim }}{\bigcirc}$ \\
\hline $\begin{array}{l}\text { Com quais os profissionais de saúde você trabalhou } \\
\text { no último ano? Você pode marcar mais de uma } \\
\text { resposta. }\end{array}$ & $\begin{array}{l}\square \text { Enfermeiro } \\
\square \text { Nutricionista } \\
\square \text { Fisioterapeuta } \\
\square \text { Farmacêutico } \\
\square \text { Terapeuta Ocupacional } \\
\square \text { Educador físico } \\
\square \text { Assistente social } \\
\square \text { Psicólogo } \\
\square \text { Dentista } \\
\square \text { Biomédico } \\
\square \text { Fonoaudiólogo } \\
\square \text { Médico }\end{array}$ \\
\hline Você cuidou de paciente com LESJ no último ano? & $\underset{\text { Simo }}{\text { Sim }}$ \\
\hline
\end{tabular}

De quantos pacientes você cuidou no último ano?

\begin{tabular}{ll}
\hline Você já cuidou de algum paciente com LESJ durante & $\bigcirc$ Yes \\
sua vida profissional? & $\bigcirc$ No
\end{tabular}

\begin{tabular}{ll}
\hline Você já utilizou alguma(s) ferramenta(s) & $\square$ SLEDAI \\
específica(s) para avaliar a atividade da doença em & $\square$ SLEDAI-2K \\
pacientes com LESJ em sua prática clínica? Você & $\square$ SELENA-SLEDAI \\
pode marcar mais de uma resposta. & $\square$ BILAG \\
& $\square$ Avaliação Global pelo médico \\
& $\square$ Avaliação Global pelo paciente/pais \\
& $\square$ Eu não conheço essas ferramentas \\
& $\square$ Nenhuma
\end{tabular}

Que outras ferramentas você já utilizou em sua prática clínica em pacientes com LESJ? Você pode marcar mais de uma resposta.

$\square$ Systemic Lupus International Collaborating Clinics/ACR damage index (SLICC/ACR-DI)

$\square$ Simple Measure of the Impact of Lupus Erythematosus in Youngsters (SMILEY)

$\square$ Childhood Health Assessment Questionnaire (CHAQ)

Childhood Health Questionnaire (CHQ)

$\square$ Eu não conheço essas ferramentas

$\square$ Nenhuma 
Confidential

Page 3

Quais dos cuidados de suporte abaixo estavam disponíveis em seu serviço no último ano? Você pode marcar mais de uma resposta.

$\square$ Protetor Solar

$\square$ Orientação para atividade física

Abordagem pela equipe multidisciplinar

Controle da pressão arterial

Orientação da função sexual

Uso de Contraceptivos

$\square$ Plano de transição para um profissional de

saúde do adulto

$\square$ Eu não conheço estes cuidados de suporte gerais

Você já observou alguns desses problemas em

pacientes com LESJ? Você pode marcar mais de uma resposta.

Má aderência medicamentosa

Hospitalização

$\square$ Consumo de cigarro

Consumo de álcool

Uso de drogas ilícitas

Câncer

Gravidez

$\square$ Não Observei nenhum desses problemas

Qual foi a estratégia para transição de cuidados ao

ambulatório adulto recomendado em seu serviço no

último ano para pacientes adolescentes e/ou com LESJ?

$\square$ Transferência baseada somente na idade

Transição clínica para adultos no mesmo serviço

Transição para reumatologista adulto no mesmo serviço

$\square$ Transição para reumatologista adulto em outro serviço

Não há programa estabelecido

$\square$ Desconheço o processo de transição de meu serviço 


\section{Confidential}

Quais foram as práticas clínicas que você

realizou/orientou aos seus pacientes no geral e/ou com LES durante o último ano? Você pode marcar mais de uma resposta.
Prontuário Eletrônico

Hospital Dia

Prescrição Medicamentosa

Chamadas telefônicas aos pacientes para lembrar suas consultas

Chamadas telefônicas para avaliações clínicas

Agendamento de consultas

Auxiliar para os recursos da comunidade

Cuidado Paliativo

Fisioterapia

Orientação Nutricional

Tratamento Odontológico

Reabilitação

Fonoaudiologia

Coleta de exames laboratoriais

Administração de injeção intrarticular

Administração de terapia intravenosa

Participação em estudos clínicos

Aconselhamento do paciente/família sobre a doença

Educação do paciente/família sobre a doença

Verificação de aderência medicamentosa

Aderência às consultas

Realizar/ensinar atividades físicas

Mensuração de sinais vitais

Avaliação de dados antropométricos

Avaliação da caderneta de vacinação

Questionários relacionados a qualidade de vida

Avaliação da dor

Monitoramento de eventos adversos

Informação do paciente para outros

profissionais/departamentos

Conhecimento do tratamento

Avaliação do paciente e cuidado clínico

Experiência em reumatologia

Boa comunicação

Habilidade para trabalhar com uma equipe

multiprofissional

Abordagem familiar

Aderência ao tratamento

Recursos financeiros

Cuidado ao paciente e à família

$\square$ Outras:

Outras:

Com qual frequência você participa de atualizações

Semestral

e congressos?

Anual

Não vou a congressos

Outra:

Outra:

Quais são os principais desafios os pacientes com

LES] em seu serviço e em seu país em geral? 


\section{Referências}




\section{Referências}

1. Vidotto JP, Pereira LA, Braga AL, Silva CA, Sallum AM, Campos LM, Martins LC, Farhat SC. Atmospheric pollution: influence on hospital admissions in paediatric rheumatic diseases. Lupus. 2012;21:526-33.

2. Nukumizu LK, Barros PC, Liphaus BL, Sallum AM, Silva CA. Sobreposição de dermatomiosite juvenil e lúpus eritematoso sistêmico. Rev Bras Reumatol. 2001;42:407-10.

3. Silva CA. Lúpus eritematoso sistêmico juvenil. Rev Bras Reumatol. 2006;46:267.

4. Marks SD, Tullus K. Autoantibodies in systemic lupus erythematosus. Pediatr Nephrol. 2012;27:1855-68.

5. Zhang L, Luan W, Geng S, Ye S, Wang X, Qian L, Ding Y, Li T, Jiang A. Lack of patient education is risk factor of disease flare in patients with systemic lupus erythematosus in China. BMC Health Serv Res. 2019;19:378.

6. Denardo BA, Tucker LB, Miller LC, Szer IS, Schaller JG. Demography of a regional pediatric rheumatology patient population. Affiliated Children's Arthritis Centers of New England. J Rheumatol. 1994;21:1553-61.

7. Gottlieb BS, llowite NT. Systemic lupus erythematosus in children and adolescents. Pediatr Rev. 2006;22:323-30.

8. Klein-Gitelman M, Reiff A, Silverman ED. Systemic lupus erythematosus in childhood. Rheum Dis Clin North Am. 2002;28:561-77.

9. Mina R, Brunner HI. Pediatric Lupus - Are there differences in presentation, genetics, response to therapy, and damage accrual compared with adult lupus? Rheum Dis Clin N Am. 2010;36:53-80.

10. Lotito AP, Campos LM, Dias MH, Silva CA. Reflex sympathetic dystrophy. J Pediatr(Rio J). 2004;80:159-62.

11. Rabelo-Júnior CN, Facó MM, Lotito APN, Silva CA. Púrpura palpável como manifestação clínica inicial do lúpus eritematoso sistêmico juvenil mimetizando púrpura de Henoch-Schönlein. Rev Paul Pediatr. 2005;23:208-13.

12. Sampaio MC, Silva CA, Pereira RM, Oliveira ZN. Lúpus eritematoso discóide na infância. Rev Paul Pediatr. 2007;25:167-71.

13. Fernandes EC, Savioli C, Siqueira JT, Silva CA. Oral health and the masticatory system in juvenile systemic lupus erythematosus. Lupus. 2007;16:713-9. 
14. Cavalcante EG, Guissa VR, Jesus AA, Campos LM, Sallum AM, Aikawa NE, Silva CA. Stevens-Johnson syndrome in a juvenile systemic lupus erythematosus patient. Lupus. 2011;20:1439-41.

15. Silva CA. Doenças refratárias ao tratamento convencional: como proceder? (Parte 2). Lúpus eritematoso sistêmico Juvenil: nefrite e envolvimento neuropsiquiátrico. Rev Bras Reumatol. 2004;44(Supl 1):41-3.

16. von Scheven E, Bakkaloglu A. What's new in paediatric SLE? Best Pract Res Clin Rheumatol. 2009;23:699-708.

17. Campos LM, Liphaus BL, Silva CA, Pereira RM. Osteoporosis in childhood and adolescence. J Pediatr(Rio J). 2003;79:481-8.

18. Pons-Estel GJ, Catoggio LJ, Cardiel MH, Bonfa E, Caeiro F, Sato E, Massardo L, Molina-Restrepo JF, Toledano MG, Barile-Fabris LA, Amigo MC, AcevedoVásquez EM, Abadi I, Wojdyla D, Alarcón-Riquelme ME, Alarcón GS, PonsEstel BA; GLADEL. Lupus in Latin-American patients: lessons from the GLADEL cohort. Lupus. 2015;24:536-45.

Gomes RC, Silva MF, Kozu K, Bonfá E, Pereira RM, Terreri MT, Magalhães CS, Sacchetti SB, Marini R, Fraga M, Carvalho LM, Barbosa CM, Carneiro-Sampaio M, Silva CA. Features of 847 Childhood-Onset Systemic Lupus Erythematosus Patients in Three Age Groups at Diagnosis: A Brazilian Multicenter Study. Arthritis Care Res (Hoboken). 2016;68:1736-41.

19. Gomes RC, Silva MF, Kozu K, Bonfá E, Pereira RM, Terreri MT, Magalhães CS, Sacchetti SB, Marini R, Fraga M, Carvalho LM, Barbosa CM, Carneiro-Sampaio M, Silva CA. Features of 847 Childhood-Onset Systemic Lupus Erythematosus Patients in Three Age Groups at Diagnosis: A Brazilian Multicenter Study. Arthritis Care Res (Hoboken). 2016;68:1736-41.

20. Novak GV, Molinari BC, Ferreira JC, Sakamoto AP, Terreri MT, Pereira RMR, Saad-Magalhães C, Aikawa NE, Campos LM, Len CA, Appenzeller S, Ferriani VP, Silva MF, Oliveira SK, Islabão AG, Sztajnbok FR, Paim LB, Barbosa CM, Santos MC, Bica BE, Sena EG, Moraes AJ, Rolim AM, Spelling PF, Scheibel IM, Cavalcanti AS, Matos EN, Robazzi TC, Guimarães LJ, Santos FP, Silva CT, Bonfá E, Silva CA; Brazilian Childhood-onset Systemic Lupus Erythematosus Group. Characteristics of 1555 childhood-onset lupus in three groups based on distinct time intervals to disease diagnosis: a Brazilian multicenter study. Lupus. 2018;27(10):1712-1717 
21. Len CA, Terreri MT, Hilário MOE. Lupus eritematoso sistêmico e infecção. Rev Bras Reumatol. 2002;42:218-22..

22. Facó MM, Nukumizu LA, Moraes AJP, Barros PCB, Troster EJ, Silva CA. Avaliação dos óbitos e necrópsias em pacientes internados em um Serviço de Reumatologia Pediátrica por um período de 10 anos. Rev Bras Reumatol. 2005;45:55-63.

23. Facó MM, Leone C, Campos LM, Febrônio MV, Marques HH, Silva CA. Factors associated to death in patients hospitalized with juvenile systemic lupus erythematosus. Braz J Med Biol Res. 2007;40:993-1002.

24. Canova EG, Rosa DC, Vallada MG, Silva CA. Invasive aspergillosis in juvenile systemic lupus erythematosus. A clinico-pathologic case. Clin Exp Rheumatol. 2002;20:736.

25. Neves PS, Facó MM, Sallum AME, Campos LMA, Rossi Júnior A, Silva CA. Herpes zoster em pacientes com lúpus eritematoso sistêmico juvenil. Rev Bras Reumatol. 2007;47:135-9.

26. Appenzeller S, Vinet E, Clarke A. Disseminated herpes zoster causing extensive skin necrosis. Lupus. 2009;18:476.

27. Silva MF, Ribeiro AS, Fiorot FJ, Aikawa NE, Lotito AP, Campos LM, Mauad T, Silva CA. Invasive aspergillosis: a severe infection in juvenile systemic lupus erythematosus patients. Lupus. 2012;21:1011-6.

28. Silva MF, Ferriani MP, Terreri MT, Pereira RM, Magalhães CS, Bonfá E, Campos LM, Okuda EM, Appenzeller S, Ferriani VP, Barbosa CM, Ramos VC, Lotufo S, Silva CA. A Multicenter Study of Invasive Fungal Infections in Patients with Childhood-onset Systemic Lupus Erythematosus. J Rheumatol. 2015;42:2296-303.

29. Ferreira JC, Marques HH, Ferriani MP, Gormezano NW, Terreri MT, Pereira RM, Magalhães CS, Campos LM, Bugni V, Okuda EM, Marini R, Pileggi GS, Barbosa CM, Bonfá E, Silva CA. Herpes zoster infection in childhood-onset systemic lupus erythematosus patients: a large multicenter study. Lupus. 2016;25:754-9.

30. Marques VL, Gormezano NW, Bonfá E, Aikawa NE, Terreri MT, Pereira RM, Magalhães CS, Guariento A, Appenzeller S, Ferriani VP, Barbosa CM, Ramos VC, Lotufo S, Silva CA. Pancreatitis Subtypes Survey in 852 Childhood-Onset 
Systemic Lupus Erythematosus Patients. J Pediatr Gastroenterol Nutr. 2016;62:328-34.

31. Ferriani MP, Silva MF, Pereira RM, Terreri MT, Saad Magalhães C, Bonfá E, Pastorino AC, Carolina Dos Santos M, Appenzeller S, Ferriani VP, Len CA, Sallum AM, Libório J, Monteiro de Castro TC, Silva CA. Chronic Spontaneous Urticaria: A Survey of 852 Cases of Childhood-Onset Systemic Lupus Erythematosus. Int Arch Allergy Immunol. 2015;167:186-92.

32. Lube GE, Ferriani MP, Campos LM, Terreri MT, Bonfá E, Magalhães CS, Aikawa NE, Piotto DP, Peracchi OA, Dos Santos MC, Appenzeller S, Ferriani VP, Pereira RM, Silva CA. Evans Syndrome at Childhood-Onset Systemic Lupus Erythematosus Diagnosis: A Large Multicenter Study. Pediatr Blood Cancer. 2016;63:1238-43.

33. Kahwage PP, Ferriani MP, Furtado JM, de Carvalho LM, Pileggi GS, Gomes FH, Terreri MT, Magalhães CS, Pereira RM, Sacchetti SB, Marini R, Bonfá E, Silva CA, Ferriani VP. Uveitis in childhood-onset systemic lupus erythematosus patients: a multicenter survey. Uveitis in childhood-onset systemic lupus erythematosus patients: a multicenter survey. Clin Rheumatol. 2017;36:547- 53.

34. Sakamoto AP, Silva CA, Ferriani MP, Pereira RM, Bonfá E, Saad-Magalhães C, Okuda E, Appenzeller S, Gomes FH, Cunha AL, Salume MH, Piotto DP, Terreri MT. Characterization of chronic arthritis in a multicenter study of 852 childhoodonset systemic lupus erythematosus patients. Rheumatol Int. 2016;36:1641-8.

35. Novak GV, Marques M, Balbi V, Gormezano NW, Kozu K, Sakamoto AP, Pereira RM, Terreri MT, Magalhães CS, Guariento A, Sallum AM, Marini R, Ferriani VP, Barbosa CM, de Castro TC, Ramos VC, Bonfá E, Silva CA. AntiRO/SSA and anti-La/SSB antibodies: Association with mild lupus manifestations in 645 childhood-onset systemic lupus erythematosus. Autoimmun Rev. 2017;16:132-5.

36. Lopes SRM, Gormezano NWS, Gomes RC, Aikawa NE, Pereira RMR, Terreri MT, Magalhães CS, Ferreira JC, Okuda EM, Sakamoto AP, Sallum AME, Appenzeller S, Ferriani VPL, Barbosa CM, Lotufo S, Jesus AA, Andrade LEC, Campos LMA, Bonfá E, Silva CA; Brazilian Childhood-onset Systemic Lupus Erythematosus Group. Outcomes of 847 childhood-onset systemic lupus erythematosus patients in three age groups. Lupus. 2017;26:996-1001.

37. Ferreira JCOA, Trindade VC, Espada G, Morel Z, Bonfá E, Magalhães CS, Silva 
CA. Epidemiology and management practices for childhood-onset systemic lupus erythematosus patients: a survey in Latin America. Clin Rheumatol. 2018;37:3299-307.

38. Castrejón I, Tani C, Jolly M, Huang A, Mosca M. Indices to assess patients with systemic lupus erythematosus in clinical trials, long-term observational studies, and clinical care. Clin Exp Rheumatol. 2014;32(5 Suppl 85):S-85-95.

39. Castrejón I, Rúa-Figueroa I, Rosario MP, Carmona L. Clinical composite measures of disease activity and damage used to evaluate patients with systemic lupus erythematosus: A systematic literature review. Reumatol Clin. 2014;10:309-20.

40. Harris PA, Taylor R, Thielke R, Payne J, Gonzalez N, Conde JG. Research electronic data capture (REDCap)--a metadata-driven methodology and workflow process for providing translational research informatics support. $J$ Biomed Inform. 2009;42:377-81.

41. Dantas CN, , Santos VEP, Tourinho FSV. A Consulta de Enfermagem como Tecnologia do Cuidado à Luz dos Pensamentos de Bacon e Galimberti. Texto Contexto Enferm. 2016;25(1):e2800014.

42. Mikdashi J, Nived O. Measuring disease activity in adults with systemic lupus erythematosus: the challenges of administrative burden and responsiveness to patient concerns in clinical research. Arthritis Res Ther. 2015;17:183.

43. Moorthy LN, Saad-Magalhães C, Sato JO, Len CA, Vasco MB, Appenzeller S, Marini R, Oliveira SK, Rodrigues M, Sztajnbok F, Almeida RG, Jesus $A A$, Campos LM, Silva CA, Peterson MG, Hassett AL, Weiss E, Verma $S$, Dahodwala MQ, Lehman TJ. Validation of the Portuguese Simple Measure of Impact of Lupus Erythematosus in Youngsters (SMILEY) in Brazil. Lupus. 2013;22:190-7.

44. Jones JT, Carle AC, Wootton J, Liberio B, Lee J, Schanberg LE, Ying J, Morgan DeWitt E, Brunner HI. Validation of Patient-Reported Outcomes Measurement Information System Short Forms for Use in Childhood-Onset Systemic Lupus Erythematosus. Arthritis Care Res (Hoboken). 2017;69:133-42.

45. Farinha F, Freitas F, Águeda A, Cunha I, Barcelos A. Concerns of patients with systemic lupus erythematosus and adherence to therapy - a qualitative study. Patient Prefer Adherence. 2017;11:1213-9.

46. Wang Y, Zhao R, Gu C, Gu Z, Li L, Li Z, Dong C, Zhu J, Fu T, Gao J. The 
impact of systemic lupus erythematosus on health-related quality of life assessed using the SF-36: a systematic review and meta-analysis. Psychol Health Med. 2019;24:978-91.

47. Bauernfeind B, Aringer M, Prodinger B, Kirchberger I, Machold K, Smolen J, Stamm T. Identification of relevant concepts of functioning in daily life in people with systemic lupus erythematosus: A patient Delphi exercise. Arthritis Rheum. 2009;61:21-8.

48. Zhang L, Luan W, Geng S, Ye S, Wang X, Qian L, Ding Y, Li T, Jiang A. Lack of patient education is risk factor of disease flare in patients with systemic lupus erythematosus in China. BMC Health Serv Res. 2019;19:378.

49. Zhang L, Geng S, Qian L, Ye S, Wang X, Lu G, Ding Y, Li T. Multidisciplinary care in patients with systemic lupus erythematosus: a randomized controlled trial in China. Int J Clin Pharm. 2019;41:1247-55.

50. dos Santos SCD, Thiengo PCS, Gallasch CH, Pires AS, Gomes HF, Junior EFP. Principais cuidados de enfermagem aos pacientes portadores de Lúpus Eritematoso Sistêmico: relato de experiência. Revista Pró-UniverSUS. 2019;10 :39-47.

51. American Academy of Pediatrics; American Academy of Family Physicians; American College of Physicians; Transitions Clinical Report Authoring Group, Cooley WC, Sagerman PJ. Supporting the health care transition from adolescence to adulthood in the medical home. Pediatrics. 2011;128:182-200.

52. Crowley R, Wolfe I, Lock K, McKee M. Improving the transition between paediatric and adult healthcare: a systematic review. Arch Dis Child. 2011;96:548-53.

53. Cramm JM, Strating MMH, Nieboer AP. The role of team climate in improving the quality of chronic care delivery: a longitudinal study among professionals working with chronically ill adolescents in transitional care programmes. BMJ Open. 2014;4:e005369.

54. Canadian Paediatric Society. Transition to adult care for youth with special health care needs. Paediatr Child Health. 2007;12:785-8.

55. Pastura PSVC, Paiva CG de. Transição dos cuidados de pacientes com doenças crônicas da pediatria para a medicina de adultos: práticas de um hospital terciário no Brasil. Rev Ped SOPERJ. 2018;18:3-10.

56. Anelli CG, Amorim ALM, Osaku FM, Terreri MT, Len CA, Reiff A. Challenges in 
transitioning adolescents and young adults with rheumatologic diseases to adult Care in a Developing Country - the Brazilian experience. Pediatr Rheumatol Online J. 2017;15:47.

57. Morisky DE, Green LW, Levine DM. Concurrent and predictive validity of a selfreported measure of medication adherence. Med Care. 1986;24:67-74.

58. Ahluwalia J, Marsch A. Photosensitivity and photoprotection in patients with lupus erythematosus. Lupus. 2019;28:697-702..

59. Oliveira DS, Hillal JPD, Cordova DDP, de Araujo DB. The lupus patient traveller: a guide to the rheumatologist. Lupus. 2019;28:1690-8.

60. World Health Organization (WHO). Definition of palliative care. [cited 5 August 2020]. Available from: https://www.who.int/news-room/factsheets/detail/palliative-care

61. Pilegi GS, Brenol Cv, da Mota LMH, Valadares LD de V, Rezende RPV, Valim V. Vacinação no paciente com doenças reumáticas. In Shinjo, SK, Moreira C. Livro da Sociedade Brasileira de Reumatologia $2^{\underline{a}}$ ed. Barueri: Manole; 2021. p.668-75.

62. World Health Organization. World health statistics 2018: monitoring health for the SDGs, sustainable development goals. Geneva: World Health Organization; 2018. (Licence: CC BY-NC-SA 3.0 IGO).

63. Ling N, Lawson E, von Scheven E. Adverse pregnancy outcomes in adolescents and young women with systemic lupus erythematosus: a national estimate. Pediatr Rheumatol Online J. 2018;16:26.

64. Teng YKO, Bredewold EOW, Rabelink TJ, Huizinga TWJ, Eikenboom HCJ, Limper M, Fritsch-Stork RDE, Bloemenkamp KWM, Sueters M. An evidencebased approach to pre-pregnancy counselling for patients with systemic lupus erythematosus. Rheumatology (Oxford). 2018;57:1707-20. 